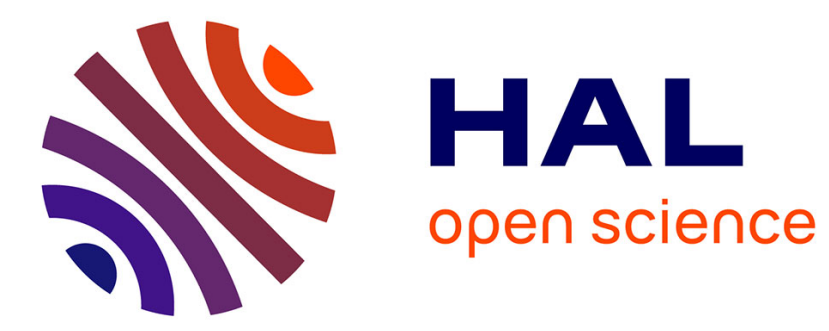

\title{
Modeling of Doppler-shifted terrestrial VLF transmitter signals observed by DEMETER
}

\author{
M. J Starks, T. F Bell, R. A Quinn, U. S Inan, D. Piddyachiy, Michel Parrot
}

\section{To cite this version:}

M. J Starks, T. F Bell, R. A Quinn, U. S Inan, D. Piddyachiy, et al.. Modeling of Doppler-shifted terrestrial VLF transmitter signals observed by DEMETER. Geophysical Research Letters, 2009, 36 (12), 10.1029/2009GL038511 . insu-03218239

\section{HAL Id: insu-03218239 \\ https://hal-insu.archives-ouvertes.fr/insu-03218239}

Submitted on 5 May 2021

HAL is a multi-disciplinary open access archive for the deposit and dissemination of scientific research documents, whether they are published or not. The documents may come from teaching and research institutions in France or abroad, or from public or private research centers.
L'archive ouverte pluridisciplinaire HAL, est destinée au dépôt et à la diffusion de documents scientifiques de niveau recherche, publiés ou non, émanant des établissements d'enseignement et de recherche français ou étrangers, des laboratoires publics ou privés. 


\title{
Modeling of Doppler-shifted terrestrial VLF transmitter signals observed by DEMETER
}

\author{
M. J. Starks, ${ }^{1}$ T. F. Bell, ${ }^{2}$ R. A. Quinn, ${ }^{3}$ U. S. Inan, ${ }^{2}$ D. Piddyachiy, ${ }^{2}$ and M. Parrot ${ }^{4}$ \\ Received 7 April 2009; revised 15 May 2009; accepted 22 May 2009; published 18 June 2009.
}

[1] Observations of signals from a terrestrial very low frequency (VLF) transmitter made by the DEMETER spacecraft inside the plasmasphere are modeled using a three-dimensional wave propagation code. The simulation results agree well with the satellite measurements, predicting both the incidence and frequency offset of Doppler-shifted signals resulting from non-ducted interhemispheric propagation paths through the plasmasphere. The observed Doppler shifts are similar to those which can result from linear mode coupling as VLF transmitter signals scatter from small-scale plasma density irregularities. Thus care must be taken to differentiate the two effects when studying the power loss of VLF waves through the ionosphere. The agreement shown between predictions and observation demonstrates the utility of the models used for understanding the wave energy distribution in the plasmasphere from terrestrial transmitters. Citation: Starks, M. J., T. F. Bell, R. A. Quinn, U. S. Inan, D. Piddyachiy, and M. Parrot (2009), Modeling of Dopplershifted terrestrial VLF transmitter signals observed by DEMETER, Geophys. Res. Lett., 36, L12103, doi:10.1029/ 2009GL038511.

\section{Introduction}

[2] One of the main goals of space weather research is to understand the dynamics of the energetic particles which comprise the radiation belts, in particular the source and loss mechanisms which control the number and lifetime of those particles in the belts at any given time. It is commonly believed [Abel and Thorne, 1998; Millan and Thorne, 2007] that the lifetimes of energetic radiation belt electrons (100 keV-1.5 MeV) on L shells in the range 1.3-2.8 are strongly influenced by VLF signals from powerful ground-based transmitters. However, this conclusion is based upon wave propagation models that have never been tested in detail. Starks et al. [2008] examined the performance of several existing VLF propagation models and the recently-developed Air Force Research Laboratory VLF Propagation Code, concluding that all of the simulations substantially overpredict wave fields in the plasmasphere from terrestrial transmitters. In the present work, we instead test the VLF Propagation Code's modeled wave normal outputs using plasma wave data from the DEMETER spacecraft.

\footnotetext{
${ }^{1}$ Space Vehicles Directorate, Air Force Research Laboratory, Kirtland AFB, New Mexico, USA.

${ }^{2}$ STAR Lab, Stanford University, Stanford, California, USA.

${ }^{3}$ AER Corporation, Lexington, Massachusetts, USA.

${ }^{4}$ LPC2E, Centre National de la Recherche Scientifique, Orleans, France.
}

Copyright 2009 by the American Geophysical Union. 0094-8276/09/2009GL038511
[3] DEMETER is the first of the Myriade series of microsatellites developed by the French National Center for Space Studies (CNES). The spacecraft was placed in a sun-synchronous polar orbit with $98.3^{\circ}$ inclination at an altitude of $710 \mathrm{~km}$ in June, 2004. The payload includes an electric field instrument (ICE) for plasma wave observations [Cussac et al., 2006] which can observe plasma waves in four separate frequency channels: DC/ULF $(0-15 \mathrm{~Hz}), \mathrm{ELF}$ $(15 \mathrm{~Hz}-1 \mathrm{kHz}), \mathrm{VLF}(15 \mathrm{~Hz}-17.4 \mathrm{kHz})$, and HF $(10 \mathrm{kHz}-$ $3.175 \mathrm{MHz})$. In the VLF channel, one component of the wave electric field is sampled at $40 \mathrm{kHz}$ and digitized with 16 bits [Berthelier et al., 2006]. In "survey" mode, twosecond spectrograms with $20 \mathrm{~Hz}$ frequency resolution are downlinked. These are supplemented by the $40 \mathrm{kHz}$ sampled data in "burst" mode. Although the nominal upper cutoff frequency of the VLF channel is $17.4 \mathrm{kHz}$, the response of this channel at $20 \mathrm{kHz}$ is only $11 \mathrm{~dB}$ below its response at $10 \mathrm{kHz}$. Thus plasma wave observations at frequencies up to $20 \mathrm{kHz}$ in this channel are routinely performed.

[4] Very strong signals at frequencies as high as $22 \mathrm{kHz}$ can also appear in the VLF channel through aliasing. In particular, $21.4 \mathrm{kHz}$ signals from the U.S. Navy VLF communications transmitter NPM $\left(20.4^{\circ} \mathrm{N},-158.2^{\circ} \mathrm{E}\right.$, $423 \mathrm{~kW}$ ) are commonly observed on DEMETER. The VLF channel response at $21.4 \mathrm{kHz}$ is approximately $10 \mathrm{~dB}$ below its response at $20 \mathrm{kHz}$, and the signals appear aliased to $18.6 \mathrm{kHz}$. Recent observations by DEMETER of signals from NPM have revealed waves with substantial frequency shifts from the aliased transmitter signal. In this paper, we show through propagation modeling that these offset signals arise from the Doppler-shifting of NPM transmissions that have propagated interhemispherically in a non-ducted fashion, and discuss their implications for the plasmaspheric VLF environment.

\section{VLF Propagation Modeling}

[5] The Air Force Research Laboratory's VLF Propagation Code [Starks et al., 2008] was employed to model DEMETER observations of the NPM VLF transmitter near Lualualei, Hawaii. The code combines a modular threedimensional ray tracer with a power flux estimation technique that includes divergence, focusing and resonant damping. When provided with suitable initial conditions, the code can predict the power flux and wave normal vector distribution in the plasmasphere due to terrestrial VLF transmitters.

[6] A magnetic meridional section of predicted plasmaspheric power flux due to the NPM transmitter is shown in Figure 1, taken through the transmitter longitude. A few features of the power distribution are particularly relevant to 


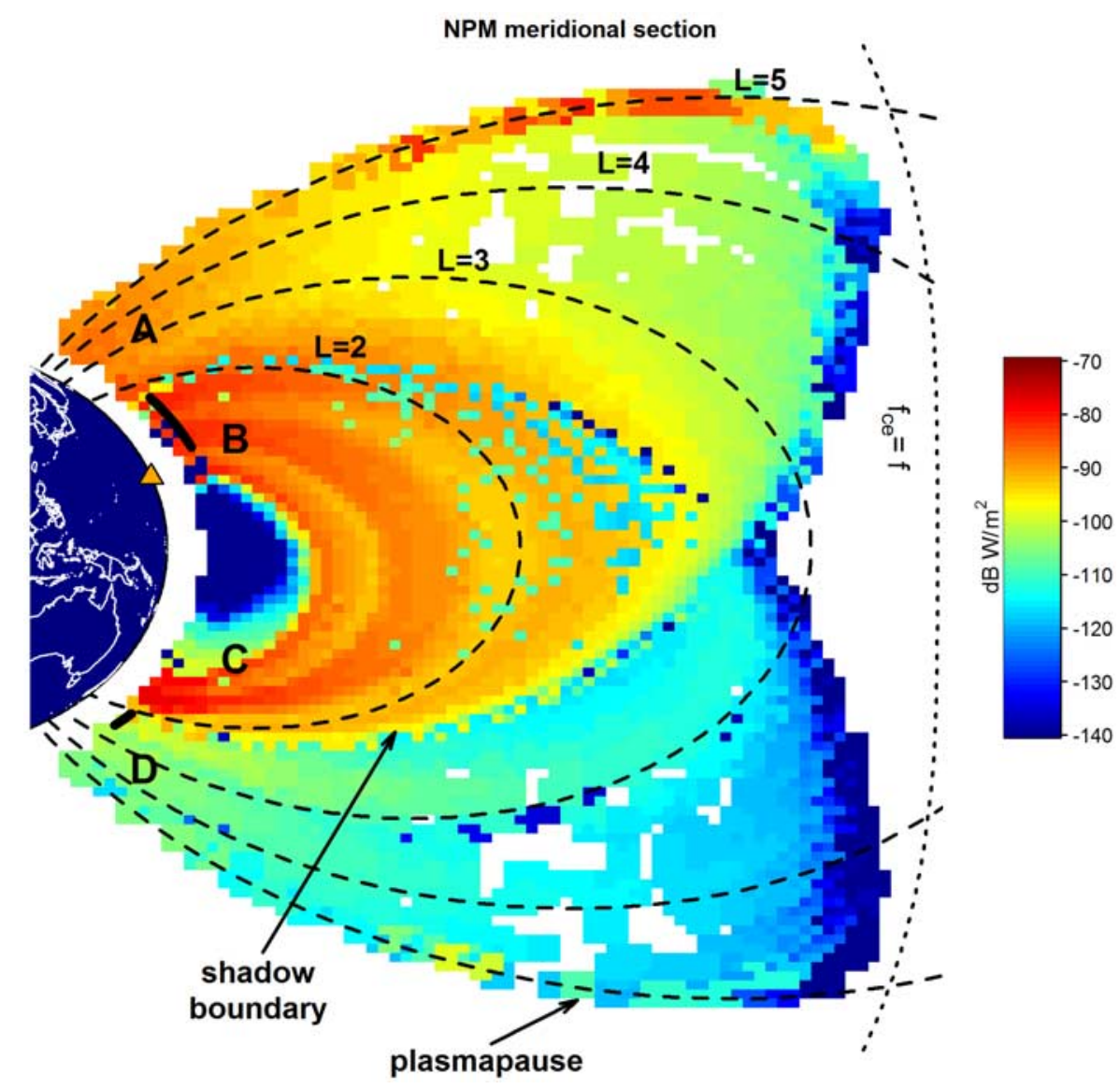

Figure 1. Meridional section of power flux predicted by AFRL's VLF Propagation Code in the plasmasphere due to NPM transmissions. The transmitter is marked by a triangle. Note the prominent shadow boundary in the conjugate hemisphere. An analogous boundary (not visible) exists in the transmitter hemisphere. The trajectories of the two DEMETER passes described here are shown as heavy black lines near the Earth. Note their proximity to the two shadow boundaries.

this study. The shadow boundary indicated by an arrow on Figure 1 prominently demarcates the region beyond which the powerful direct signals from the transmitter hemisphere do not reach. The presence of a caustic where the wave frequency matches the electron gyrofrequency causes upgoing rays to bend and form the boundary. In the process, the wave normals of the waves rotate to become highly oblique to the background magnetic field. There is an analogous shadow boundary formed by rays propagating upward from the hemisphere conjugate to the transmitter. Because such rays are substantially weaker, the boundary is not visible in Figure 1. Its location is entirely symmetrical to that of the transmitter hemisphere shadow boundary.

[7] Based on the model outputs, a low-Earth orbiting (LEO) spacecraft observing the NPM transmitter within the plasmasphere will encounter four different situations. At location $\mathrm{A}$ in Figure 1, there will be a strong signal from upgoing waves originating near the transmitter, but no waves coming from the conjugate hemisphere. At location $\mathrm{B}$, those strong upgoing signals will be accompanied by weak waves from the conjugate hemisphere having highly oblique wave normals, caused by the long propagation path through the plasmasphere. At location $\mathrm{C}$, the situation is reversed, and it is the strong waves from the transmitter hemisphere which have traveled the long path, while the rays from the conjugate hemisphere are coming directly up at the spacecraft. Location D is beyond the transmitter hemisphere's shadow boundary, and only weak upgoing conjugate signals should be detected.

[8] Typically, multiple signals from a transmitter arriving along different paths are separated using timing information. Because NPM was being keyed during the DEMETER observations, this is certainly possible. However, we can better distinguish signals of different origins by recognizing that waves with different wave normal angles should have different Doppler shifts when observed aboard a moving spacecraft according to the relation:

$$
f_{\text {recv }}=f_{\text {xmit }} \cdot[1-(\vec{n} \bullet \vec{v}) / c]
$$

where $f_{\text {recv }}$ is the frequency received aboard the moving satellite, $f_{x m i t}$ is the frequency of the fixed terrestrial transmitter, $n$ is the wave refractive index vector of the received signal, $v$ is the satellite vector velocity, and $c$ is the vacuum speed of light.

[9] For a circular orbit like that of DEMETER, Doppler shifting should only occur for waves with wave normals oriented away from the radial direction, i.e., where $\vec{n} \bullet \vec{v}$ is non-zero. Thus waves propagating directly up toward the spacecraft should have minimal frequency offset, while those taking the long path through the plasmasphere should show a significant Doppler shift, visible in spectrograms as traces offset from the $18.6 \mathrm{kHz}$ frequency band. 
04 April 2006: Electric Field Intensity
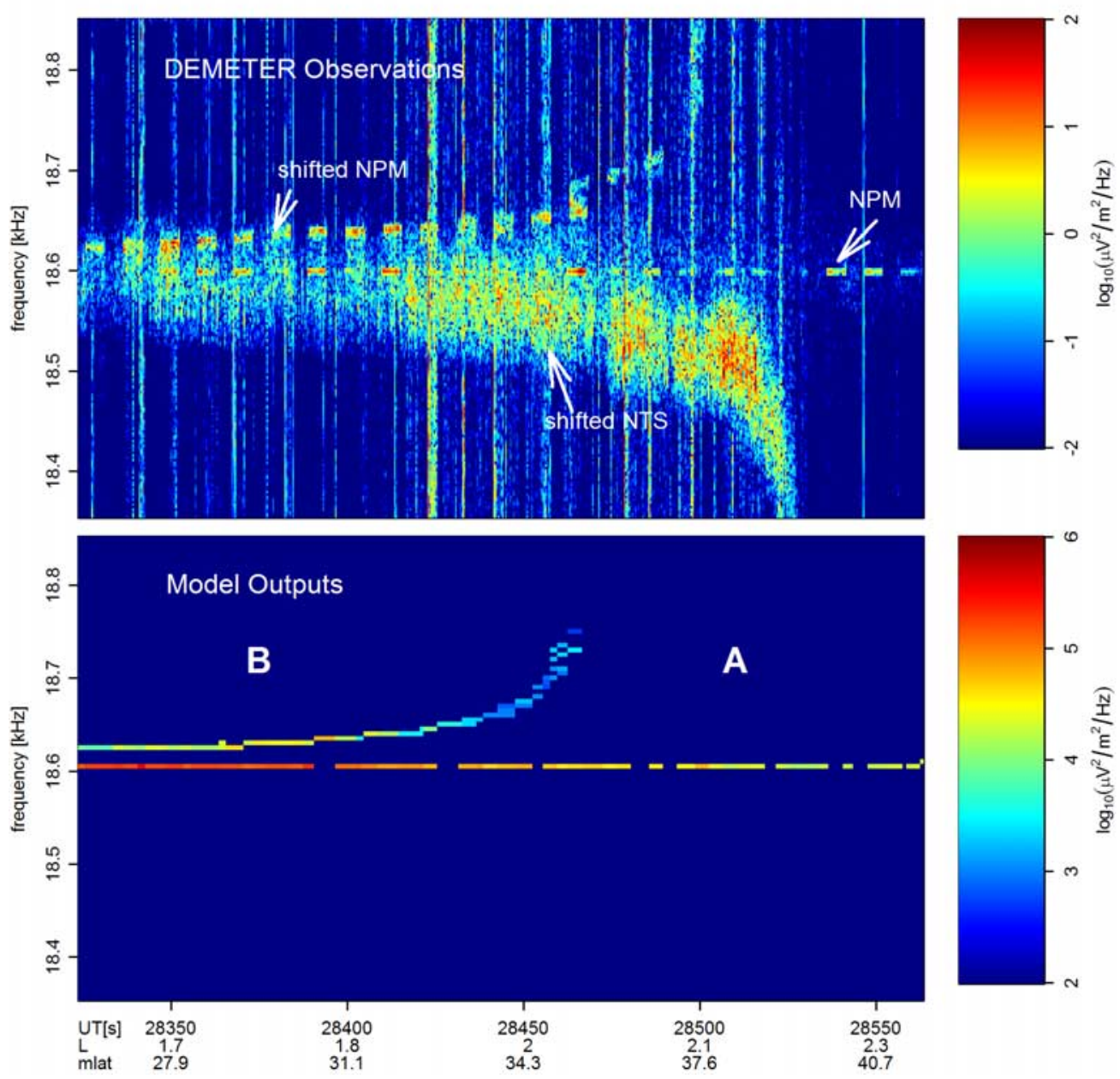

Figure 2. Spectrograms for a DEMETER pass near the shadow boundary in the transmitter hemisphere (regions from Figure 1 are indicated). (top) DEMETER data; (bottom) model outputs. Time is in seconds after midnight UT. Notice the transmitter waves propagating directly up to the satellite are aliased to $18.6 \mathrm{kHz}$ and are relatively powerful. The weaker, shifted waves come from the conjugate hemisphere. The predicted and observed Doppler shifts largely agree, although the edge of the shadow boundary occurs at lower latitude in the model than in the data. The broad, negatively-shifted feature in the data is not modeled, and gaps in Figure 2 (bottom) result from insufficient ray coverage, not an attempt to reproduce the keying.

[10] This is indeed the case for many DEMETER observations of NPM, and we next present representative cases where the spacecraft transited the $\mathrm{AB}$ and $\mathrm{CD}$ boundaries in Figure 1. The trajectories of the two low-altitude $(670 \mathrm{~km})$ passes are shown by heavy lines in Figure 1.

\section{DEMETER Observations and Model Predictions}

[11] Figure 2 shows a DEMETER observation during which the spacecraft passed from region $\mathrm{B}$ into region $\mathrm{A}$ near the NPM magnetic meridian. Figure 2 (top) shows a frequency-time spectrogram of the measured plasma wave electric field intensity. The signals in Figure 2 arise from two separate transmitters, the NPM transmitter in Hawaii and the VLF communications transmitter NTS near Woodside, Victoria, Australia. NTS operates on $18.6 \mathrm{kHz}$, with a bandwidth of approximately $50 \mathrm{~Hz}$. This comparatively wide bandwidth gives it a noise-like quality in the spectrogram.
[12] Notice the keyed narrow-band $21.4 \mathrm{kHz}$ NPM signals aliased to $18.6 \mathrm{kHz}$; these have propagated essentially directly upward through the ionosphere to the spacecraft and as a result have nearly radial wave normal angles (and hence no Doppler shift). Notice also the offset replica of the NPM signals apparent at higher frequencies. These waves have propagated from the transmitter to the conjugate hemisphere through the Earth-ionosphere waveguide, penetrated into the plasmasphere, and traveled across the magnetic equator to the spacecraft. In the process, their wave normal angles have become highly oblique, resulting in a Doppler shift to the observed signal of $25-50 \mathrm{~Hz}$. The actual amount of shift increases as DEMETER approaches the conjugate shadow boundary, until the offset waves disappear entirely, marking the transition from region $\mathrm{B}$ to region $\mathrm{A}$.

[13] The noise-like signals from the Australian transmitter have also propagated from the conjugate region through the plasmasphere to DEMETER and consequently exhibit a significant Doppler shift as DEMETER approaches the conjugate shadow boundary. Since the location of the 
01 February 2006: Electric Field Intensity

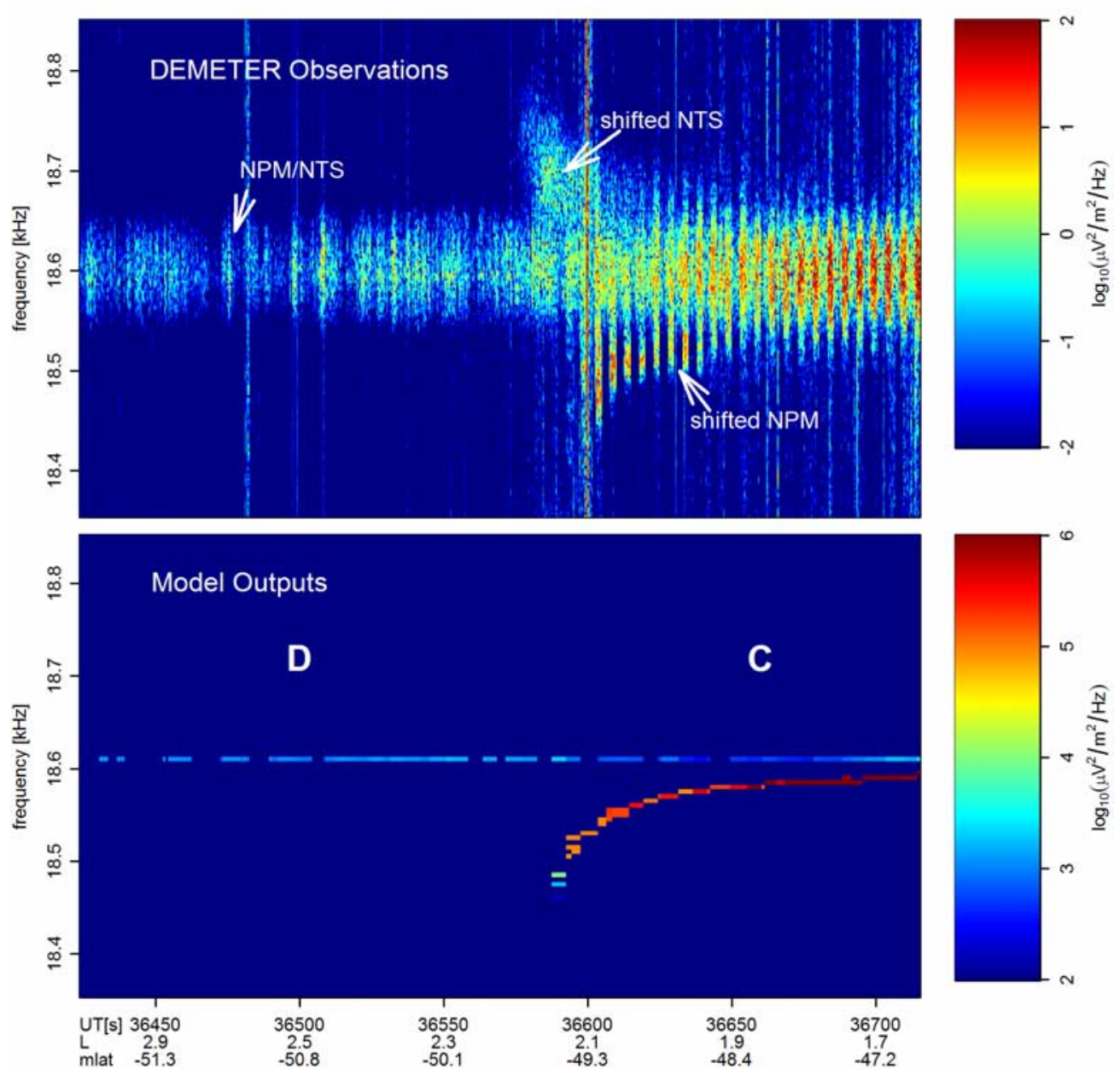

Figure 3. Same as Figure 2, but for a pass in the conjugate hemisphere. The directly ascending transmitter signals are weaker in this hemisphere, and the Doppler shift is negative. Good agreement is achieved for the frequency shift magnitude, and the edge of the shadow boundary is predicted more accurately.

shadow boundary is a function of frequency, the Australian transmitter signals cut off at higher latitude than those of NPM. The Doppler shift of these signals is negative, as would be expected since their wave vectors point northward and DEMETER is moving northward. The Doppler shift of the $21.4 \mathrm{kHz}$ NPM signals is also negative, but appears positive in Figure 2 because of aliasing.

[14] Figure 2 (bottom) shows the predictions from AFRL's VLF Propagation Code, described in detail by Starks et al. [2008]. The code combines a modular threedimensional ray tracing program with algorithms that estimate power. Rays are traced from initial points spread over a 97,200-point equal-area grid defined within the ionosphere and with intensities given by the outputs of transmitter and ionospheric absorption models. No horizontal density gradients are postulated and it is assumed that due to refraction the wave normals of the upgoing rays are initially aligned with the gradient of the ionospheric density, i.e., in the radial direction. Rays are traced in the whistler mode into the plasmasphere until they re-enter the ionosphere. The three-dimensional computation permits ray paths to vary freely within and across magnetic meridians, subject only to the constraints imposed by the cold plasma dispersion relation. There are no field-aligned ducts presumed in this model. The Earth's magnetic field is represented by a tilted offset dipole [Fraser-Smith, 1987]. The ambient plasma density is generated by a multi-species diffusive equilibrium density model [Angerami and Thomas, 1964; Thomson, 1987]. Landau and cyclotron damping are computed explicitly along the ray paths using a nominal warm plasma distribution.

[15] The actual DEMETER trajectory was flown through a simulation of the NPM transmitter's plasmaspheric wave field (without keying) and the model wave electric field intensity and wave normal angle recorded. The model results were then aliased and rendered as a spectrogram for direct comparison to the spacecraft observations. The strong $18.6 \mathrm{kHz}$ aliased NPM signal is present in Figure 2 (bottom), as is the weaker Doppler-shifted copy. Note the good agreement of the frequency offset when compared to the observations, at least until the edge of the shadow boundary is approached. The observed boundary appears to be located at somewhat higher latitude than predicted.

[16] Figure 3 shows a DEMETER pass in the conjugate hemisphere, again near the NPM magnetic meridian, as the spacecraft departed region D for region C. Figure 3 (top) plots the observations. Once again the signals that appear in the figure arise from two separate transmitters, the NPM 
transmitter in Hawaii and NTS in Australia. The noise-like signals from the Australian transmitter reach DEMETER by propagating directly upward through the ionosphere.

[17] In the left portion of Figure 3 (top) we see only weak signals from both transmitters with no frequency offset. The NPM signals detected there have propagated to the conjugate region through the Earth-ionosphere waveguide, and then traveled directly upward to the spacecraft. They again have essentially radial wave normal vectors, resulting in little Doppler shift. The variability is likely due to plasma density irregularities along the propagation path. At the right side of Figure 3 (top) we do see Doppler shifted signals, but this time with much higher amplitudes than those taking the direct path. These waves originate in the transmitter hemisphere where field intensities are much higher. Their frequency offset stems from their oblique wave normals, combined with the motion of the DEMETER spacecraft.

[18] Note that the Doppler shift in this case is opposite in sign from Figure 2. In that case the wave normals had a northward component, along DEMETER's track, because they originated in the southern hemisphere. Here the waves propagate along the field line in the opposite direction, develop southward components to their wave normals, and create a Doppler shift of the opposite sign. The actual shifts are opposite of those shown in Figure 2, because of aliasing.

[19] Figure 3 (bottom) plots the simulated spectrogram based on the ray tracing model. Again the relative magnitudes and frequency offsets agree with the observations, and in this case the position of the shadow boundary is also correctly predicted.

[20] A statistical study of DEMETER measurements was performed to ascertain the prevalence of the observed agreement between model and data. In the transmitter hemisphere, the weak shadow boundary signals are expected between $\mathrm{L}=2-2.2$. There exist very little DEMETER burst mode data near NPM above $\mathrm{L}=2$, so reliance on the lower-resolution survey mode data is required. In the survey mode data, the "long path" NPM signals are usually extremely difficult to detect in the transmitter hemisphere due to the interfering NTS signal. In the conjugate hemisphere, however, the Doppler-shifted signals are much stronger, as they originate near the NPM transmitter. Out of 78 DEMETER passes in the southern hemisphere, 42 had sufficient signal to noise ratio to theoretically detect Doppler-shifted NPM signals. Of these, 41 cases were similar to Figure 3, demonstrating a clear shadow boundary with the predicted location and Doppler shift. The remaining case showed no trace of a shadow boundary, despite having low enough noise levels that one would expect to see it. In other words, for this particular data set, the model accurately predicts the DEMETER conjugate shadow boundary observations $98 \%$ of the time, when DEMETER is on the correct L-shell and the signal-tonoise ratio is sufficiently high.

[21] Finally, it is worth noting from Figure 3 that the predicted field intensities of the directly-received waves are routinely two to four orders of magnitude larger than those actually observed. Starks et al. [2008] found that the existing initial conditions models for terrestrial transmitters substantially overestimate the wave energy reaching the plasmasphere, often by $20 \mathrm{~dB}$ or more. When compared to the model outputs, the DEMETER observations presented here are consistent with those findings. The matter is not a concern for this study, however. Here we focus solely on the observed Doppler shift, which is a function of the wave normal angles in different propagation regimes, not of absolute power.

\section{Discussion and Conclusions}

[22] Spacecraft observations of Doppler shifts in VLF transmitter signals have been reported sporadically over the last few decades. One of the first reports concerned observations from the OGO-4 spacecraft of VLF transmitter signals propagating from the northern hemisphere through the plasmasphere to the spacecraft in the southern hemisphere [Walter and Angerami, 1969]. The Doppler shifts observed on OGO-4 were a few hundred Hz. More recently, Doppler shifts have been commonly observed in short wavelength quasi-electrostatic whistler mode waves generated through linear mode coupling as VLF transmitter signals scatter from planar magnetic field aligned plasma density irregularities [Bell and Ngo, 1990]. In this type of scattering a fixed frequency transmitter signal generally excites a pair of quasi-electrostatic whistler mode waves with anti-parallel wave vectors which are roughly perpendicular to the irregularity. On a moving spacecraft, the quasi-electrostatic waves appear as symmetrical sideband signals about the input signal. An example of this effect can be seen in Figure 3 beginning at the shadow boundary. The fixed frequency NPM signals appear to have a total bandwidth of roughly $150 \mathrm{~Hz}$.

[23] Observations by the DEMETER spacecraft suggest that the basic three-dimensional structure of wave energy in the plasmasphere from terrestrial VLF transmitters can be understood using AFRL's VLF Propagation Code. Frequency-resolved measurements of the NPM transmitter presented in section 3 demonstrate that the model wave normal angles are reasonably accurate, producing good predictions of the observed Doppler shift. Although DEMETER orbits below $700 \mathrm{~km}$ altitude, the shifted signals have their origins in the conjugate hemisphere and have traveled very long distances through the plasmasphere, crossing many magnetic L-shells. Hence, the DEMETER observations are able to provide validation of the model performance not only near $700 \mathrm{~km}$, but over the entire plasmaspheric propagation path.

[24] These results also indicate that the primary cause of large frequency offsets in LEO observations of terrestrial VLF transmitters is simple Doppler shift rather than more complicated plasma processes, such as ducting or absorption and re-radiation. In addition, they support the work of Clilverd et al. [2008] who concluded that NPM signals primarily propagate in a non-ducted fashion.

[25] Finally, this work demonstrates that quasi-electrostatic (highly oblique) waves may arise along the shadow boundary from simple non-ducted propagation, and that such waves are readily apparent aboard the DEMETER satellite. They may also be generated by scattering from plasma density irregularities, appearing as sidebands near the source wave, and this has been suggested [Bell et al., 2008] as the fate of some of the "missing" power reported by Starks et al. [2008] in the plasmasphere from terrestrial 
VLF transmitters. The combined DEMETER and modeling work indicates that care must be taken to distinguish these two possible origins when identifying quasi-electrostatic sideband waves associated with the source transmitter electromagnetic wave.

[26] Acknowledgments. The Stanford University STAR Laboratory is a Guest Investigator of the French micro-satellite DEMETER operated by CNES (Centre National d'Etudes Spatiales). The Stanford authors were supported by the Department of the Air Force, under grant F49620-03-10338-P00002 and contract F19628-03-C-0059-P00002, and by the Office of Naval Research under Prime Award N000140710789 to the University of Maryland with subcontract Z882802 to Stanford University.

\section{References}

Abel, B., and R. M. Thorne (1998), Electron scattering loss in Earth's inner magnetosphere: 1. Dominant physical processes, J. Geophys. Res., 103, $2385-2396$

Angerami, J. J., and J. O. Thomas (1964), Studies of planetary atmospheres: 1. The distribution of electrons and ions in the Earth's exosphere, J. Geophys. Res., 69, 4537-4560.

Bell, T. F., and H. D. Ngo (1990), Electrostatic lower hybrid waves excited by electromagnetic whistler mode waves scattering from planar magneticfield-aligned plasma density irregularities, J. Geophys. Res., 95, $149-172$.

Bell, T. F., U. S. Inan, D. Piddyachiy, P. Kulkarni, and M. Parrot (2008), Effects of plasma density irregularities on the pitch angle scattering of radiation belt electrons by signals from ground based VLF transmitters, Geophys. Res. Lett., 35, L19103, doi:10.1029/2008GL034834.

Berthelier, J. J., et al. (2006), ICE, the electric field experiment on DEMETER, Planet. Space Sci., 54, 456-471.
Clilverd, M. A., C. J. Rodger, R. Gamble, N. P. Meredith, M. Parrot, J.-J. Berthelier, and N. R. Thomson (2008), Ground-based transmitter signals observed from space: Ducted or nonducted?, J. Geophys. Res., 113, A04211, doi:10.1029/2007JA012602.

Cussac, T., M.-A. Clair, P. Ultre-Guerard, F. Boisson, G. Lassalle-Ballier, M. Ledu, C. Elisabelar, X. Passot, and N. Rey (2006), The DEMETER microsatellite and ground segment, Planet. Space Sci., 54, 413-427.

Fraser-Smith, A. C. (1987), Centered and eccentric geomagnetic dipoles and their poles, 1600-1985, Rev. Geophys., 25, 1-16.

Millan, R. M., and R. M. Thorne (2007), Review of radiation belt relativistic electron loss, J. Atmos. Sol. Terr. Phys., 69, 362-377.

Starks, M. J., R. A. Quinn, G. P. Ginet, J. M. Albert, G. S. Sales, B. W. Reinisch, and P. Song (2008), Illumination of the plasmasphere by terrestrial very low frequency transmitters: Model validation, J. Geophys. Res., 113, A09320, doi:10.1029/2008JA013112.

Thomson, N. R. (1987), Ray-tracing the paths of very low latitude whistlermode signals, J. Atmos. Terr. Phys., 49, 321-338.

Walter, F., and J. J. Angerami (1969), Nonducted mode of VLF of the conjugate hemispheres: Observations on OGO's 2 and 4 of the 'WalkingTrace' whistler and of Doppler shifts in fixed frequency transmissions, J. Geophys. Res., 74, 6325-6370.

T. F. Bell, U. S. Inan, and D. Piddyachiy, STAR Lab, Stanford University, 350 Serra Mall, Stanford, CA 94305-9515, USA.

M. Parrot, LPC2E, Centre National de la Recherche Scientifique, 3A Avenue de la Recherche Scientifique, F-45071 Orleans CEDEX 2, France.

R. A. Quinn, AER Corporation, 131 Hartwell Avenue, Lexington, MA 02421-3136, USA.

M. J. Starks, Space Vehicles Directorate, Air Force Research Laboratory, 3550 Aberdeen Avenue SE, Kirtland AFB, NM 87117-5776, USA. (michael.starks@us.af.mil) 